\title{
The quantum strategy of completeness: On the self-foundation of mathematics
}

\author{
Vasil Penchev, vasildinev@gmail.com \\ Bulgarian Academy of sciences: Institute of Philosophy and Sociology: \\ Dept. of Logical Systems and Models
}

\begin{abstract}
Gentzen's approach by transfinite induction and that of intuitionist Heyting arithmetic to completeness and the self-foundation of mathematics are compared and opposed to the Gödel incompleteness results as to Peano arithmetic. Quantum mechanics involves infinity by Hilbert space, but it is finitist as any experimental science. The absence of hidden variables in it interpretable as its completeness should resurrect Hilbert's finitism at the cost of relevant modification of the latter already hinted by intuitionism and Gentzen's approaches for completeness. This paper investigates both conditions and philosophical background necessary for that modification. The main conclusion is that the concept of infinity as underlying contemporary mathematics cannot be reduced to a single Peano arithmetic, but to at least two ones independent of each other. Intuitionism, quantum mechanics, and Gentzen's approaches to completeness an even Hilbert's finitism can be unified from that viewpoint. Mathematics may found itself by a way of finitism complemented by choice. The concept of information as the quantity of choices underlies that viewpoint. Quantum mechanics interpretable in terms of information and quantum information is inseparable from mathematics and its foundation.
\end{abstract}

Key words: axiom of choice, finitism, Gentzen proof of completeness, Gödel incompleteness theorems, Heyting arithmetic, Hilbert program, Peano arithmetic, quantum mechanics, completeness of quantum mechanics

\section{INTRODUCTION}

Cantor's set theory involved actual infinity. However many paradoxes directly or indirectly linked to the concept of infinite set were found. One of the first was Russell's (Russell 1902).

Hilbert suggested his program for the foundation of set theory and all mathematics on arithmetic (Hilbert 1931; Kreisel 1958). Thus the "consistent part of actual infinity" in set theory should be reduced to that of finite natural numbers as the auxiliary notion of any finite number having some property if any that exists, i.e. utilizing the " $\varepsilon$-symbol" (Hilbert, Bernays 1970), or "potential infinity" seen as any indefinite finiteness.

Nevertheless, Gödel (1931) has managed to show that neither any formal system containing Peano (1889) arithmetic neither the arithmetic itself can be both complete and consistent.

In fact, Peano (1889)'s axioms had inspired (Gunderson 2011: 22; Peano 1889: 5) by Dedekind (1888)'s foundation of the natural numbers on set theory and especially on the wellordering of infinite set $^{1}$. Thus a kind of "historical vicious circle" turned out to have been constituted: well-ordering in set theory should serve as the foundation of set theory and "actual infinity" by the meditation of Peano arithmetic.

\footnotetext{
${ }^{1}$ Dedekind different terms and definitions and the correspondence to ours can be only incomplete.
} 
Mathematical induction is crucial among Peano's axioms: It is neither provable in type theory (Royse 1969) nor explanatory (Lange 2009).

Furthermore, at least two different enough types of consistent proofs immediately referring to the consistency of Peano arithmetic appeared (Gentzen 1936; 1938; Smorynski 1973; Kanckos 2010). Gentzen's one was in the framework of classical approach shared by Hilbert and Gödel only utilizing transfinite induction while the others admitted for the "excluded third" to be suspended in the way of intuitionism as to infinite sets. Both were interpreted by Gödel in his works (Gödel [1938]; Gödel 1958: Tait 2005; 2010).

Almost at the same time, a physical and thus experimental science, quantum mechanics met the problem of completeness for the hypothesis of the so-called hidden variables (Einstein, Podolsky, Rosen 1935). In fact, it as well as Schrödinger's study (also 1935) forecast the phenomena of entanglement on the base of Hilbert space.

A few theorems (Neumann 1932: 167-173; Kochen and Specker 1968) were deduced from the mathematical formalism of quantum mechanics, namely the infinitely dimensional complex Hilbert space. They show that any hidden variables cannot exist in quantum mechanics, and thus it is complete.

Bell (1964) demonstrated how the hypothesis of hidden variable might be tested experimentally. The corresponding experiments (Clauser, Horne 1974; Aspect, Grangier, Roger 1981; 1982) and many, many others after that categorically show that no hidden variables in quantum mechanics, and consequently it is complete.

In fact, the completeness of quantum mechanics both involving infinity by Hilbert space and being finitist as any experimental science should resurrect Hilbert's finitism at the cost of relevant modification already hinted by intuitionism and Gentzen's approach for completeness.

This paper investigates both conditions and philosophical background necessary for that modification.

It is organized as follows:

Section I discusses the valid range of Gödel's negative result to Hilbert's program.

Section II specifies the method of investigation involving both opposite concepts of Gödel mathematics and Hilbert mathematics.

Section III investigates the difference in Gödel's and Gentzen's approach to be obtained opposite results about completeness.

Section IV interprets Gentzen's approach to infinity as the factual independence of two finitenesses or a single but two-dimensional finiteness, and thus the representability of set theory by two independent Peano arithmetics.

Section V shows that intuitionist approach to completeness is consistent and even equivalent to that interpretation.

Section VI introduces the completeness of quantum mechanics in terms of that of arithmetic and set theory and therefore of mathematics interpreting Hilbert space as a kind of generalization of Peano arithmetic. 
At last, Section VII considers the question whether a kind of neo-Pythagoreanism is implied as philosophical background both of "Hilbert mathematics" and quantum mechanics and information and even not less as to "Gödel mathematics", and thus to mathematics at all.

\section{BOUNDARIES OF GÖDEL'S INCOMPLETENSS}

The key for the validity of Gödel (1931)'s result to Hilbert's program is within the former itself and even within its core: that statement $[R(q) ; q]$ stating to be false.

Its approach can be described as skeptical to finitism (Resnik 1974: 145), but it can be referred to itself generating a kind of skepticism about the skepticism in turn.

Furthermore Hilbert's program can be relevantly modified after re-estimating the meaning of Gödel incompleteness (Murawski 1999: 303-323).

Gödel has written (1931: 175 [footnote 14]) that any antinomy, e.g. that of Liar (even mentioned expressly by Gödel ibidem), can serve to construct the proof of incompleteness.

If the statement $[R(q) ; q]$ is allowed to be referred to itself, this would be a direct contradiction, and any theory containing that would be useless being unable to differ "true" from "false". Thus the self-reference in the most of set-theory paradoxes, forced by the concept of actual infinity, implies possibly inconsistency.

Gödel built in his work a context for the statement $[R(q) ; q]$, in which the level and meta-level to be reliable and consistent separate from each other. Then the statement $[R(q) ; q]$ would not be self-referent and contradictory or Gödel's proof containing it.

Gödel's proof depends crucially on the disjunctive distinction between level and metalevel in the statement $[R(q) ; q]$. However if that kind of distinction is made in the most of the set-theory paradoxes themselves, they would not be self-contradictory, too. This is an observation utilized by Russell in his theory of types (Principia [both editions]: 63[I]; 60[II]).

Also vice versa: one can question whether one cannot construct any artificial context, in which the so-called Gödel first incompleteness theorem (Satz VI) to be forced to refer to itself therefore stating its own incompleteness.

The idea can be illustrated by "Liar": Normally "I lie" is used to use in the context "I lie that ' $A$ ". The paradox is generated only if and only after " $A$ ' has been identified as "I lie", which is not a normal use categorically distinguishing the level of "A' from the meta-level of "I lie". If one says only "I lie", it can be interpreted as "I lie that "I lie"". Then, "I lie" as used separately is identified with "I lie' in that "I lie that " $A$ ". The Liar paradox is generated.

One can "unfold" the Liar paradox into Yablo (1993)'s paradox: Both share the "fixed point structure" (Priest 1997: 242).

One can say that their pair demonstrates a possible approach to the simile of selfreferentiality and infinity or at least that of self-referential finiteness and infinity. A next step in the same way will be explicated in the present paper: the fundamental simile of two independent "finitinesses", to which a single and self-referential finiteness can be always decomposed, and infinity. Thus in particular, the Liar and Yablo paradox can be consider as the discrete and continuous version of one and the same paradox. 
Furthermore Yablo's paradox can be related furthermore to sorites paradox or to "Achilles and the torture": Always a leap is necessary to passes into any different quality. Then if one suggests the "relativeness of the concept of set" (Skolem [1922]) and therefore not only that of different "inifinities", but not less also that of finiteness and infinity ${ }^{2}$, the transition between finiteness and infinity will need an analogical jump. "The discrete in the continuous" features mathematics also according to Browser (Stigt, 1979: 403).

The next step is the following conjecture: two finitenesses supplied by any jump between them are equivalent to the pair of finiteness and infinity rather than only similar.

Meaning these considerations furthermore, one can repeat the stages necessary to construct the Liar paradox as a little above already as to "Satz VI" normally referring to any other theory under its conditions but not to itself ever:

It satisfies its conditions as they are described in it. Then "Satz VI" can be isolated and therefore it is forced to be referred to itself.

At last, "Satz VI" in relation to any other theory under its conditions is to be identified with "Satz VI" isolated and therefore forced to refer to itself.

Consequently "The Liar" is transformed almost literally into a modified paradox of "The Stating incompleteness" with a very important difference: "The Liar" generates inconsistency, but "The Stating incompleteness" generates only incompleteness.

In the same context and technique, one can put the problems about the Gödel number of "Satz VI", of the undecidability of the "Satz VI" and therefore the proof's constructiveness, and a few others.

\section{HILBERT MATHEMATICS VS GÖDEL MATHEMATICS AND THE PHILOSOPHICAL APPROACH IN MATHEMATICS}

Incompleteness after "The Stating Incompleteness" unlike the Liar inconsistency implies that the corresponding statement as well as its negation is able to be considered as an independent postulate. So the incompleteness of mathematics in the sense of "Satz VI" closely linked to the disjunctive distinction of the level and meta-level should be accepted only as an option depending on a relevant axiom as what might be interpreted the "Satz VI" itself. It would be meta-mathematical in essence and would divide mathematics in two forms according to either the acceptance or the rejection of that axiom.

Let us designate the former as "Gödel mathematics" and the latter as "Hilbert mathematics" and describe them:

"Gödel mathematics": The level and meta-level are disjunctively divided and therefore mathematics is incomplete in principle for the level of mathematics cannot include the meta-level (e.g. that of reality) in principle remaining forever incomplete for that impossibility.

\footnotetext{
2 "Sogar die Begriffe "endlich", "unendlich", "einfach unendliche Reihe" usw. werden nur relativ innerhalb der axiomatischen Mengenlehre" (Skolem 1970[1922]: 143).
} 
"Hilbert mathematics": The level and meta-level merge and penetrate into each other. Mathematics is able to be complete and therefore to self-found. It cannot be distinguished from reality where "reality" is used as a generalizing term for any meta-level.

The practice in mathematical research, proofs, and publications show that any hypothesis can be perfected further and further in the most cases by means of additional and more precise definitions and specifications.

This process is able to be seen formally in terms of intuitionism as follows:

Two "creative subjects" representable as two Heyting arithmetics (Krivtsov 1999) play a zero sum game consisting in proving theorems in favor correspondingly of Gödel mathematics or of Hilbert mathematics. Any move represents further definitions and specifications so that a new theorem either in Gödel mathematics or in Hilbert mathematics is proved therefore sharing all course of game of both "creative subjects" before the last move.

If the game is equitable, and the players are independent of each other, the game is able to be always continued or to be a draw.

Being at the viewpoint of that game, or respectively at the judge's one, can be an example of philosophical approach in mathematics unlike each properly mathematical approach granting a single player's one by granting one or more relevant axioms for identifying with one of the players.

The discussed completeness and therefore the self-foundation of mathematics would be trivial in Hilbert mathematics as being postulated.

The next sections should be placed in the framework of Gödel mathematics.

\section{GÖDEL'S INCOMPLETENESS VS GENTZEN'S COMPLETENESS}

One can suppose that the Gödel incompleteness originates from the deficiency of the mathematical structure, on which mathematics should be grounded. However, that deficiency can imply two alternative and maybe equivalent ways for proving completeness:

- Qualitative deficiency: some other mathematical structure rather than arithmetic is necessary: e.g. geometry;

- Quantitative deficiency: arithmetic but more than one is what is needed: e.g. at least two ones.

One can utilize a kind of more than an analogy to the so-called fundamental theorem of algebra that every non-constant single-variable polynomial with complex coefficients has at least one complex root: It needs a more general structure than the real numbers, namely the field of complex numbers, for which it can be proved.

Analogically, the self-foundation of mathematics needs some more general structure than the positive integers in order to be provable.

The difference in the conditions of Gödel's incompleteness and Gentzen's completeness is crucial (Horská 2014). In other words, the problem is to be localized that specific element, to which the opposite result in each of both case is due: 
As the primitive recursive functions in Gentzen (1936 and 1938)'s approach are equivalent to Peano arithmetic being just all possible calculations in Peano arithmetic, the single added condition is transfinite induction in comparison to Gödel's opposite result.

Furthermore, transfinite induction cannot be proved in Peano arithmetic (Gentzen 1943) therefore being an independent additional condition.

Consequently, the conjecture is that just transfinite induction should be somehow understood in terms of Peano arithmetic though it cannot be reduced to it. That is the sense of the interpretation in the next section.

\section{ANOTHER INTERPRETATION OF GENTZEN'S COMPLETENESS}

Transfinite induction can be relevantly defined even within Peano arithmetic (Sommer 1995) as well as ordinals and the corresponding fixed points investigated (Jäger 1993). Thus nonstandard models are also definable in it (Ikeda, Tsuboi 2007).

Gentzen himself linked his approach for proving completeness to Hilbert's finitism (Sieg 2012; Gentzen 1954) and intuitionism (Gentzen 1974; 1974a).

Transfinite induction can be seen as a second and necessarily different induction, but rather similar to the usual mathematical induction in the Peano axioms:

The former continues "after" the latter from a conventional least transfinite ordinal designated usually as $\omega$, but just as mathematical induction where 1 is interpreted as $\omega$. Then transfinite induction might be interpreted as two independent mathematical inductions and thus of two independent Peano arithmetics as any mathematical induction implies a Peano arithmetic in order to be able to be defined. The second Peano arithmetic would be the single condition, by which Gödel's incompleteness can be differed from Gentzen's completeness.

There are at least a few approaches for the second and independent Peano arithmetic to be introduced:

1. It is able to be merely postulated as such.

2. It can be intentionally distinguished from the first one so: There is no one-to-one mapping of $n$ to $n+1$ for the first Peano arithmetic, but there is that (of $\omega+n$ to $\omega+n+1$ ) for the second one.

3. The first one refers to natural numbers, and the second one refers to ordinals or to wellorderings of infinite sets, to which all infinite sets can be ordered eventually in virtue of the axiom of choice.

The sense of that interpretation of transfinite induction as to infinity is the following: Infinity is merely a second finiteness absolutely independent of the first one. The gap between those two dimensions of finiteness is able to represent exhaustedly the entire meaning of infinity for mathematics.

In turn the sense of the same gap can be alternatively represented as the suspension of the "excluded third" as to infinite sets in Heyting arithmetic.

\section{INTUITIOTIONIST COMPLETENESS AND ITS INTERPRETTAION BY GÖDEL}


The well-orderings of transfinite induction can be rather naturally linked to intuitionist theories (Howard, Kreisel 1966; Scarpellini 1969; 1972; Friedman, Ščedrov 1985). Bar induction can be seen as a two dimensional induction in virtue of the bar theorem ${ }^{3}$ (Kleene, Vesley 1965: 43-51). Bar induction added to constructive ZFC can prove a kind of consistency of set theory (Rathjen 2006).

The second-dimensional Peano arithmetic copes with the argument of diagonalization (Montagna 1980) restoring it from one-dimensional Gödel's use to the initial two-dimensional Cantor's use.

The mechanism of generating that gap in Heyting arithmetic for allowing of the "third" as to an infinite set of natural numbers is the following:

Granting any infinite set of natural numbers, there exists always a gap of the "third" between it and its complementation to all natural numbers. Consequently, the former and latter can be directly interpreted as the first and second Peano arithmetic for Gentzen's completeness by transfinite induction as above.

Indeed Heyting arithmetic is also complete in an exact sense (Visser 1982; Kanckos 2010) just as Gentzen's approach to Peano arithmetic can be discussed as a proof of completeness.

The so-called Dialectica interpretation of Heyting arithmetic by Gödel (1958) is analogical in a sense.

\section{GÖDEL INCOMPLETENESS VS THE COMPLETENESS OF QUANTUM MECHANICS: GÖDEL AND EINSTEIN}

Gödel and Einstein are both Jews, refugees from Nazism, and even close friends in Princeton (Yourgrau 2006). However, Gödel came to Princeton in 1940. The famous triple article of Einstein, Podolsky, and Rosen "Can Quantum-Mechanical Description of Physical Reality Be Considered Complete?” was published in 1935. So, there hardly existed a direct influence, but rather a shared style of thought and even some common mathematical structure underlying the problem of completeness both in the foundation of mathematics and in quantum mechanics.

Indeed the complex Hilbert space implies the absence of hidden variables (Neumann 1932: 167-173; Kochen, Specker 1968) and thus the completeness of quantum mechanics.

One can demonstrate that the approach of quantum mechanics is related to that of Gentzen or of intuitionism to arithmetic and the foundation of mathematics:

First, the complex Hilbert space involves infinity at least in two independent and even symmetric ways:

1. It is infinitely dimensional: It can be discussed as a generalization of Peano arithmetic and even as two of those together with its dual space coinciding with it identically. Indeed it (or "both") can be represented as an infinite series of qubits, in each of which can be "recorded" two complex numbers $\left(\alpha\right.$ and $\beta$ ) such that $|\alpha|^{2}+|\beta|^{2}=1$. If any qubit being a unit ball is shrunk to a point, and conventionally granting $\alpha=0$ and $\beta=1$, a Peano arithmetic will be obtained. So,

\footnotetext{
${ }^{3}$ The two-dimensionality can be contrasted on the background of the one-dimensionality of the corresponding theorem (König 1927) in mathematics based on set theory.
} 
its "rough structure" is representable as two complementary and therefore independent, but identical Peano arithmetics.

2. Each of its "axes" is infinite, two: It can be discussed as a generalization of the usual three-dimensional Euclidean geometry ${ }^{4}$ where furthermore any axis is complex and representable as a qubit. Thus it unlike the real Hilbert space does not suggest any well-ordering within any axis by itself, but only in virtue of the axiom of choice. So involving also its "fine structure", the complex Hilbert space is thinkable as a doubled countable set of uncountable continua.

3. If the axiom of choice is applied, any qubit in turn is identifiable as the basic "rough stricture" of two independent Peano arithmetics; and vice versa: the "rough structure" without well-ordering corresponds to any qubit (or its "fine structure"): The complex Hilbert space is perfectly symmetric in a sense including the axiom of choice.

Second, what that perfect structure should describe adequately according to quantum mechanics is the system of the measured quantum system(s) and measuring device(s). Thus quantum mechanics, unlike classical one, needs a dual structure similar to that of any statistical thermodynamic theory consisting both of too many but very, very tiny microscopic elements and their large macroscopic system.

Two incommensurable finiteneses turn out to be involved in thus: the one for the set of tiny elements with the quantity of action commeasurable with the Plank constant and the other for their common system with the macroscopic measuring device(s).

\section{MATHEMATICS: COMPLETENESS VS INCOMPLETENESS}

A formal debate about the completeness of mathematics has been constructed above on the base of self-referential incompleteness of stating incompleteness.

Each step of that construction has included dividing into two as well as the division into two of that dividing itself (i.e. either the axiom of choice or not). Infinity has been able to be represented by means of that construction as to two both separated (Gentzen's approach) or inseparable (Heyting arithmetic) finitenesses (i.e. to two identical but independent Peano arithmetics). Consequently even in the "Gödel mathematics", completeness is provable by the mere doubling of Peano arithmetic equivalent to complementing it by the option of choice.

One can demonstrate that Hilbert's par excellence finitist idea about $\varepsilon$-symbol (Hilbert, Bernays 1970: 9-18) as well interpretable as partly equivalent to Gentzen's transfinite induction (Kreisel, 1958a; Zach 2003; Towsner 2005) corresponds to the complementation of Peano arithmetic with choice: The $\varepsilon$-symbol contents that choice if a given number exists or not:

Peano arithmetic is insufficient in the following sense: It should be complemented by a "white sheet" and choices for ones or other symbols of it to be able to be recorded unlimitedly on that white sheet.

The above construction of dividing into two can be found in the complex Hilbert space underlying quantum mechanics even with perfect symmetry already elucidated.

\footnotetext{
${ }^{4}$ A geometric approach to the foundation of mathematics has been an alternative at the turn of the XIX century (Tazzioli 2003).
} 
Furthermore, the construction of dividing including the division of that dividing itself can be exhaustedly represented by the concepts of information and choice. The inseparability into two is right the case of quantum information.

The completeness of Hilbert mathematics should be interpreted in a kind of neoPythagoreanism as any reality external to it and separable from it does not exist: All is mathematics.

On the contrary, Gödel mathematics to complete itself should reproduce the external reality within itself as a second by identical mathematics grounded on a second identical and independent Peano arithmetic whether in the way of Gentzen or in that of intuitionism, or in that of Hilbert's $\varepsilon$-symbol.

\section{CONCLUSION AND FUTURE WORK}

Anyway mathematics should be complete and groundable in terms of finitism complemented by choice and representable by the underlying concept of information as the quantity of choices.

Quantum mechanics interpretable in terms of information and quantum information is inseparable from mathematics and its foundation. Thus mathematics turns out to be a whole with the physical world, too. 


\section{REFERENCES:}

Aspect, A., P. Grangier, and G. Roger (1981) "Experimental Tests of Realistic Local Theories via Bell's Theorem,“ Physical Review Letters 47 (7): 460-463.

Aspect, A., P. Grangier, and G. Roger (1982) "Experimental Realization of Einstein-PodolskyRosen-Bohm Gedanken Experiment: A New Violation of Bell's Inequalities,“ Physical Review Letters 49 (2): 91-94.

Bell, J. (1964) "On the Einstein - Podolsky - Rosen paradox," Physics (New York) 1 (3): 195-200.

Clauser, J. F. and M. A. Horne (1974) "Experimental consequences of objective local theories," Physical Review D 10 (2): 526-535

Dedekind, R. (1888) Was sind und was sollen die Zahlen? Braunschweig, Friedrich Vieweg \& Sohn.

Einstein, A., B. Podolsky, N. Rosen (1935) "Can Quantum-Mechanical Description of Physical Reality Be Considered Complete?” Physical Review 47 (10): 777-780.

Friedman, H. M., and A. Ščedrov (1985) "Arithmetic transfinite induction and recursive wellorderings," Advances in Mathematics 56 (3): 283-294.

Gentzen, G. (1936) "Die Widerspruchfreiheit der reinen Zahlentheorie," Mathematische Annalen 112 (1): 493-565.

Gentzen, G. (1938) „Neue Fassung des Widerspruchsfreiheitsbeweises für die reine Zahlentheorie,” Forschungen zur Logik und zur Grundlegung der exakten Wissenschaften, New Series 4 (1): 19-44.

Gentzen, G. (1943) "Beweisbarkeit und Unbeweisbarkeit von Anfangsfällen der transfiniten Induktion," Mathematische Annalen 119 (1): 140-161.

Gentzen, G. (1954) "Zusammenfassung von Mehreren Vollständigen Induktionen zu einer Einzigen,“ Archiv für mathematische Logik und Grundlagenforschung, 2 (1): 1-3.

Gentzen, G. (1974) "Der erste Widerspruchsfreiheitsbeweis für die klassische Zahlentheorie," Archiv für mathematische Logik und Grundlagenforschung 16 (3-4): 97-118.

Gentzen, G. (1974a) "Über das Verhältnis zwischen intuitionistischer und klassischer Arithmetik," Archiv für mathematische Logik und Grundlagenforschung 16 (3-4): 119-132.

Gödel, K. (1931) "Über formal unentscheidbare Sätze der Principia mathematica und verwandter Systeme I,“"Monatshefte für Mathematik und Physik 38 (1): 173-198.

Gödel, K. [1938] "Lecture at Zilsel's," in Collected Works (S. Feferman, J. W. Dawson, W. Goldfarb, C. Parsons, and R. M. Solovay: eds.), Vol. III, Oxford, Oxford University Press, 1995, pp. 87-113.

Gödel, K. (1958) "Über eine bisher noch nicht benützte erweiterung des finiten standpunktes," Dialectica 12 (3-4): 280-287.

Gunderson, D. S. (2011) Handbook of mathematical induction: theory and applications. Boca Raton, FL: CRC Press.

Hilbert, D. (1931) "Die Grundlegung der elementaren Zahlenlehre," Mathematische Annalen 104 (1): 485-494.

Hilbert, D. und P. Bernays (1970) Grundlagen der Mathematik II (Zweite Auflage), Berlin, Heidelberg, New York, Springer.

Horská, A. (2014) Where is the Gödel-point hiding: Gentzen's consistency proof of 1936 and his representation of constructive ordinals, Heidelberg, New York, Dordrecht, London, Springer.

Howard, W. A. and G. Kreisel. (1966) "Transfinite Induction and Bar Induction of Types Zero and One, and the Role of Continuity in Intuitionistic Analysis," The Journal of Symbolic Logic 31 (3): 325-358. 
Ikeda, K. and A. Tsuboi (2007) "Nonstandard models that are definable in models of Peano Arithmetic," Mathematical Logic Quarterly 53 (1): 27-37.

Jäger, G. (1993) "Fixed points in Peano arithmetic with ordinals," Annals of Pure and Applied Logic 60 (2): 119-132.

Kanckos, A. (2010) "Consistency of Heyting arithmetic in natural deduction," Mathematical Logic Quarterly 56 (6): 611-624.

Kleene, S. C. and R. E. Vesley (1965) The foundations of intuitionist mathematics, especially in relation to recursive functions, Amsterdam, North-Holland Publishing Company.

Kochen, S. and E. Specker (1968) "The problem of hidden variables in quantum mechanics," Journal of Mathematics and Mechanics 17 (1): 59-87.

König, D. (1927) "Über eine Schlussweise aus dem Endlichen ins Unendliche," Acta Scientarium mathematicarum (Szeged) 3 (2-3): 121-130.

Kreisel, G. (1958) "Hilbert's programme," Dialectica 12 (3-4): 346-372.

Kreisel, G. (1958a) "Mathematical Significance of Consistency Proofs," Journal of Symbolic Logic 23 (2): 155-182.

Krivtsov, V. N. (1999) "Note on extensions of Heyting's arithmetic by adding the "creative subject"," Archive for Mathematical Logic 38 (3): 145-152.

Lange, M. (2009) "Why proofs by mathematical induction are generally not explanatory," Analysis 69 (2): 203-211.

Montagna, F. (1980) "Interpretations of the first-order theory of diagonalizable algebras in Peano arithmetic," Studia Logica 39 (4): 347-354.

Murawski, R. (1999) Recursive functions and metamathematics: problems of completeness and decidability, Gödel's theorems, Dordrecht (Netherlands) Boston, Kluwer Academic.

Neumann, J. von (1932) Mathematische Grundlagen der Quantenmechanik, Berlin, Julius Springer.

Peano, I. (1889) Arithmetices principia: nova method exposita, Romae. Florentiae. Fratres Bocca.

Priest, P. (1997) "Yablo's paradox," Analysis 57 (4): 236-242.

Rathjen, M. (2006) "A note on Bar Induction in Constructive Set Theory," Mathematical Logic Quarterly 52 (3): 253 - 258.

Resnik, M. D. (1974) "On the philosophical significance of consistency proofs," Journal of Philosophical Logic 3 (1-2): 133-147.

Royse, J. R. (1969) "Mathematical induction in ramified type theory," Mathematical Logic Quarterly 15 (1-3): 7-10.

Russell, B. (1902) "Letter to Frege (16.06.1902)," in Gotlob Frege: Philosophical and mathematical correspondence (ed. G. Gabriel). Chicago: University of Chicago Press, 1980, pp. 130-131.

Scarpellini, B. (1969) "Some applications of Gentzens second consistency proof," Mathematische Annalen 181 (4): 325-344.

Scarpellini, B. (1972) "Induction and transfinite induction in intuitionistic systems," Annals of Mathematical Logic 4 (2): 173-227.

Schrödinger, E. (1935) "Die gegenwärtige Situation in der Quantenmechanik," Naturwissenschaften 23 (48): 807-812; 23 (49): 823-828; 23 (50): 844-849.

Sieg, W. (2012) "In the Shadow of Incompleteness. Hilbert and Gentzen," in (Dybjer, P., Lindström, Sten; Palmgren, Erik; Sundholm, G., eds.) Epistemology versus Ontology: essays on the philosophy and foundations of mathematics in honour of Per Martin-Löf. Dordrecht, New York, Springer, pp. 87-127. 
Skolem, T. [1922] "Einige Bemerkungen zur axiomatischen Begründung der Mengenlehre," in Selected works in logic (J. E. Fenstad). Oslo: Universitetsforlaget, 1970, pp. 137-152.

Sommer, R. (1995). "Transfinite induction within Peano arithmetic," Annals of pure and applied $\operatorname{logic} 76$ (3): 231-289.

Stigt, W. P. van (1979) "The rejected parts of Brouwer's dissertation on the foundations of mathematics, “ Historia mathematica: international journal of history of mathematics 6 (4): 385-404.

Tait, W. W. (2005) "Gödel's Reformulation of Gentzen's First Consistency Proof for Arithmetic: The No-Counterexample Interpretation," The Bulletin of Symbolic Logic 11 (2): 225-238.

Tait, W. W. (2010) "Gödel on Intuition and on Hilbert's finitism," in Kurt Gödel: essays for his centennial (S. Feferman, C. Parsons, S. Simpson, eds.), New York, Cambridge University Press, Ithaca, NY, Association for Symbolic Logic, pp. 88-108.

Tazzioli, R. (2003) "Towards a history of the geometric foundations of mathematics: Late XIXth century, “Revue de Synthèse 124 (1): 11-41.

Towsner, H. (2005) "Epsilon substitution for transfinite induction," Archive for Mathematical Logic, 44 (4): 397-412.

Smorynski, C.A. (1973) “Applications of Kripke models," in Troelstra, A. S. (ed.) Metamathematical investigation of intuitionistic arithmetic and analysis. Berlin, Heidelberg, New York, Springer, pp. 324-391.

Visser, A. (1982) "On the completenes principle: A study of provability in Heyting's arithmetic and extensions," Annals of Mathematical Logic 22 (3): 263-295.

Whitehead, N.A., and B. Russell (any of both editions) Principia mathematics, Vol. I, Cambridge, University Press.

Yablo, S. (1993) "Paradox without Self-Reference," Analysis 53 (4): 251-252.

Yourgrau, P. (2006) A World Without Time: The Forgotten Legacy of Gödel and Einstein, New York, Perseus Books Group.

Zach, R. (2003) "The Practice of Finitism: Epsilon Calculus and Consistency Proofs in Hilbert's Program," Synthese 137 (1-2): 211-259. 